\title{
Protective effects of osthole, a natural derivative of coumarin, against intestinal ischemia-reperfusion injury in mice
}

\author{
WENPENG DONG ${ }^{1 *}$, ZHEN ZHANG $^{2 *}$, ZHENGJUN LIU $^{3 *}$, HAO LIU ${ }^{3}$, XIANYUE WANG ${ }^{1}$, \\ SHENGHUI BI ${ }^{1}$, XIAOWU WANG ${ }^{1}$, TAO MA ${ }^{1}$ and WEIDA ZHANG ${ }^{1}$ \\ ${ }^{1}$ Department of Cardiovascular Surgery, Guangzhou General Hospital of Guangzhou Military Command, Guangzhou, \\ Guangdong 510010; ${ }^{2}$ Department of General Surgery, The First Affiliated Hospital of Anhui Medical University, \\ Hefei, Anhui 230022; ${ }^{3}$ Department of Vascular and Endocrine Surgery, Nanfang Hospital, \\ Southern Medical University, Guangzhou, Guangdong 510515, P.R. China
}

Received February 4, 2013; Accepted April 2, 2013

DOI: 10.3892/ijmm.2013.1347

\begin{abstract}
Intestinal ischemia/reperfusion (I/R) injury is considered to be associated with high morbidity and mortality rates. Osthole, a natural derivative of coumarin, has been shown to exert a variety of pharmacological and therapeutic effects under physiological and pathological conditions. In the present study, to investigate the protective effects of osthole against intestinal I/R injury, various doses of osthole (5, 10, 25 and $50 \mathrm{mg} / \mathrm{kg}$ ) were pre-administered to mice subjected to intestinal I/R injury. A dose-dependent increase in the survival rate was observed in the osthole-treated mice. Pre-treatment with osthole $(50 \mathrm{mg} / \mathrm{kg})$ attenuated the destruction of epithelial cells within the villi induced by intestinal I/R injury, and suppressed oxidative stress, neutrophil infiltration and modulated nitric oxide (NO) levels. Moreover, the increased I $\kappa \mathrm{B} \alpha$ phosphorylation and nuclear factor (NF)- $\kappa \mathrm{B}$ nuclear translocation induced by I/R injury were significantly decreased following pre-treatment with osthole. Taken together, our data demonstrate that osthole exerts protective effects against intestinal I/R injury in mice by suppressing oxidative stress, neutrophil infiltration and NO levels, partly through the inhibition of NF- $\kappa \mathrm{B}$ nuclear translocation. Hence, the findings of the present study provide insight into the mechanisms through which osthole exerts its protective effects against intestinal I/R injury.
\end{abstract}

\section{Introduction}

Intestinal ischemia/reperfusion (I/R) injury represents a significant clinical problem in numerous situations, such as small

Correspondence to: Dr Weida Zhang, Department of Cardiovascular Surgery, Guangzhou General Hospital of Guangzhou Military Command, No. 111 Liu Hua Road, Guangzhou, Guangdong 510010, P.R. China

E-mail: weidazhanggz@163.com

${ }^{*}$ Contributed equally

Key words: nitric oxide, osthole, reactive oxygen species bowel transplantation, cardiopulmonary bypass, acute mesenteric ischemia, abdominal aortic aneurysm surgery, intestinal obstruction, as well as hemorrhagic, traumatic and septic shock $(1,2)$. Intestinal I/R injury triggers a highly complex cascade of events resulting in decreased contractile activity, increased microvascular permeability and the dysfunction of the mucosal barrier (3-7). Although the definitive pathogenesis regarding intestinal I/ $\mathrm{R}$ injury remains obscure, the hallmarks of this condition have been well defined, including reactive oxygen species (ROS), recruitment of activated leukocytes, cytokine release, cellular apoptosis and mitochondrial dysfunction (8). ROS, the most critical effector of intestinal I/R injury, can react with all biological macromolecules (nucleic acids, proteins, carbohydrates and lipids) in a destructive manner and initiate a wide range of toxic oxidative reactions, which include the initiation of lipid peroxidation, the direct inhibition of mitochondrial respiratory chain enzymes and membrane sodium/ potassium adenosine 5 '-triphosphate-ase activity, inactivation of membrane sodium channels and other oxidative modifications of proteins $(9,10)$. In addition, the overproduction of nitric oxide (NO) generated by inducible NO synthase (iNOS) is also characterized in intestinal $\mathrm{I} / \mathrm{R}$, which aggravates intestinal oxidative stress (11). The nuclear factor (NF)- $\kappa \mathrm{B}$ pathway has been reported to play an important role in intestinal I/R which is responsible for ROS accumulation and cell apoptosis (12). Moreover, oxidative stress promotes inflammatory cell infiltration and mitochondrial dysfunction. Thus, the therapeutic strategy of removing free radicals and reducing NO overproduction may be potentially effective for protection against intestinal I/R injury.

However, to date, there is still lack of effective therapeutic treatments for intestinal I/R injury. Osthole, a natural derivative of coumarin, is an active constituent extracted from some medicinal plants (13) which has been shown to exert a variety of pharmacological and therapeutic effects. Accumulating evidence has demonstrated that osthole possesses a variety of pharmacological properties, including antitumor (14), anti-osteoporotic (15), anti-diabetic (16), anti-inflammatory (17) and anti-allergic properties (18). Recently, osthole has been demonstrated to exert therapeutic effects against cerebral ischemic stroke $(19,20)$ and cardiac hypertrophy in rats $(21)$. 
Thus, the effects of osthole on intestinal I/R injury need to be further investigated.

The aim of this study was to investigate the effect of osthole in a mouse model of intestinal $\mathrm{I} / \mathrm{R}$ and delineate the underlying mechanisms. Using a mouse model, we first observed that pre-treatment with osthole exerted protective effects on mice with intestinal I/R injury in a dose-dependent manner. Pre-treatment with osthole markedly ameliorated villus height and attenuated the destruction of epithelial cells within the villi. The effect of osthole against intestinal I/R injury was evaluated by measuring the levels of myeloperoxidase (MPO), malondialdehyde (MDA), superoxide dismutase (SOD) and glutathione (GSH). Pre-treatment with osthole exerted protective effects, including the suppression of oxidative stress, neutrophil infiltration and NO levels. Increased $\mathrm{I} \kappa \mathrm{B} \alpha$ phosphorylation and $\mathrm{NF}-\kappa \mathrm{B}$ nuclear translocation induced by I/R injury were also significantly decreased following pre-treatment with osthole. In this study, the effects of osthole in the early phase following intestinal I/R injury in mice were investigated to provide a mechanistic basis for the protective effects of osthole against intestinal I/R injury.

\section{Materials and methods}

Animals and experimental groups. Adult male BALB/c mice were obtained from the Laboratory Animal Center of the Fourth Military Medical University, Xi'an, China and housed under standard conditions. They were kept in cages at a fairly constant room temperature and were exposed to a 12/12 h light/ dark cycle. Each animal was fasted overnight prior to surgery, but had access to water ad libitum. All animal experiments were performed in accordance with the Prevention of Cruelty to Animals Act 1986, under the guidelines of the National Health and Medical Research Council for the Care and Use of Animals for Experimental Purposes in China.

The mice were randomly divided into 3 groups $(n=13$ per group): i) Sham group: all the surgical steps were performed; however, intestinal I/R was not induced. The animals were kept under anesthesia for the duration of the intestinal I/R procedure. ii) I/R group: intestinal I/R was induced and this group served as the control for the osthole-treated group. iii) Osthole group: a single dose of osthole $(50 \mathrm{mg} / \mathrm{kg}$ daily for 3 days) was injected via the tail vein. Osthole ( $>98 \%$ purity) was a gift from Dr W. Liu (Department of Neurology, Xijing Hospital, Fourth Military Medical University, Xi'an, China). The mice in each group were subdivided into 3 subgroups. The first subgroup was used for histological examination and immunofluorescence staining ( $n=4$ per group); the second subgroup was used for detecting MPO, GSH, SOD, MDA and NO levels ( $n=6$ per group); and the third subgroup was used for western blot analysis ( $n=3$ per group).

Model of intestinal I/R injury. Following an acclimation period of at least 3 days, the mice were prepared for surgery under deep sodium pentobarbital anesthesia. All procedures were performed with the animals breathing spontaneously and body temperature was maintained at $37^{\circ} \mathrm{C}$ using a water-circulating heating pad. The animals were subjected to I/R as previously described (22). Briefly, a midline laparotomy was performed. The superior mesenteric artery was identified and isolated, and the blood supply to the intestine was interrupted for $1 \mathrm{~h}$ by occlusion of the superior mesenteric artery using a microvascular clamp. Ischemia was confirmed by the loss of pulsation of the mesenteric artery and its branches or a pale color of the intestine. Following the ischemic phase, the clamp was removed to allow the restoration of the blood flow (reperfusion) for $6 \mathrm{~h}$, which was confirmed by the return of the pulses, re-establishment of the pink color and enhanced intestinal peristalsis. Once reperfusion was secured, the wound was sutured and the animal was allowed to awaken from the anesthesia. The mice were administered an overdose of pentobarbital prior to euthanasia.

Histology and tissue injury scoring. For isolation of the intestinal segments, tissue from the entire intestine was cut into 6 equal segments, of which a section of $1-1.5 \mathrm{~cm}$ at the middle of each segment was collected for histological analysis. Formalin-fixed and paraffin-embedded tissue sections were cut (4- $\mu \mathrm{m}$-thick) and stained with hematoxylin and eosin for histological examination. The above procedure was performed by a physician.

Intestinal sections from each animal were scored for mucosal damage as previously described (23). Briefly, a score of 0 was assigned to a normal villus; villi with tip distortion were scored as 1; villi lacking goblet cells and containing Guggenheims' spaces were scored as 2; villi with patchy disruption of the epithelial cells were scored as 3; villi with exposed but intact lamina propria and epithelial cell sloughing were assigned a score of 4; villi in which the lamina propria was exuding were scored as 5; and finally, villi displaying hemorrhage or denudation were scored as 6 . All histological analyses were performed by a pathologist in a blinded manner. Five random high-power fields (x200) were examined per sample.

MPO assay. The intestinal samples were weighed and homogenized in a solution prepared from the assay kit (Jiancheng Institute of Biotechnology, Nanjing, China), and homogenates were obtained and used for MPO assay. The reaction was initiated by adding hydrogen peroxide in the medium and 3,3,5,5-tetramethylbenzidine was used as an oxidizable dye to produce yellow color compounds. The optical density was recorded at $460 \mathrm{~nm}$ and the results were expressed as $\mathrm{U} / \mathrm{g}$ wet tissue.

Detection of MDA, GSH and SOD in intestinal tissue. The intestinal samples were homogenized in $0.05 \mathrm{~mol} / 1$ phosphate buffer. The homogenates were centrifuged at 4,000 rpm for $20 \mathrm{~min}$ at $4^{\circ} \mathrm{C}$ and the MDA, GSH and SOD content in the supernatant was measured using the corresponding kits (Jiancheng Institute of Biotechnology).

SOD activity and MDA levels were measured using the xanthine oxidase and thiobarbituric acid method, respectively. SOD activity was measured at $500 \mathrm{~nm}$. One SOD activity unit was defined as the enzyme amount causing 50\% inhibition in $1 \mathrm{ml}$ reaction solution/mg tissue protein and the result was expressed as U/mg protein. MDA was determined at $532 \mathrm{~nm}$ and the results were expressed as nmol/mg protein. GSH levels were measured through a reaction using dithiobisnitrobenzoic acid. GSH activity was determined at $420 \mathrm{~nm}$ and the results were expressed as $\mathrm{mg} / \mathrm{g}$ protein.

NO synthesis can contribute to nitrite accumulation. The concentration of nitrite in the intestinal samples was measured 
following a reaction with Griess reagent (sulfanilamide $1 \%$, naphthylethylene diamine $0.01 \%, \mathrm{H}_{3} \mathrm{PO}_{4} 5 \%$ ) to reflect the production of $\mathrm{NO}$ at $550 \mathrm{~nm}$. The results were expressed as $\mu \mathrm{mol} / \mathrm{g}$ protein. Detailed procedures for the above measurements were performed according to the instructions of kit. The protein content was determined using a kit based on the Bradford assay and bovine serum albumin was used as a standard.

Western blot analysis. Total protein lysates from frozen tissues were prepared in ice-cold RIPA buffer $(20 \mathrm{mM}$ HEPES $\mathrm{pH} 7.5$, $150 \mathrm{mM} \mathrm{NaCl}, 1 \mathrm{mM}$ EDTA, $10 \%$ glycerol, $0.5 \%$ sodium deoxycholate, $1 \%$ Nonidet P-40, $0.1 \%$ SDS and protease inhibitor cocktails). Nuclear extracts were prepared using a Nuclear Protein Extraction kit (Beyotime Biotechnology, Shanghai, China). Protein samples were immunoblotted

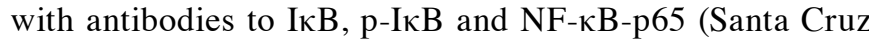
Biotechnology, Inc., Santa Cruz, CA, USA), and the proteinantibody immune complexes were detected with horseradish peroxidase-conjugated secondary antibodies and enhanced chemiluminescence reagents (Pierce Biotechnology, Rockford, IL, USA). For detection, an ECL chemiluminescence system (GE Healthcare, Piscataway, NJ, USA) was used. Signals were detected and quantified using the LAS-3000 image analyzer (FujiFilm, Tokyo, Japan).

Immunofluorescence staining. Staining was performed on slides of 4- $\mu \mathrm{m}$-thick sections from intestinal tissue. Following deparaffinization and dehydration, the slides were immersed in $10 \mathrm{mM}$ sodium citrate buffer containing $0.05 \%$ Tween-20 (pH 6.0) for $5 \mathrm{~min}$ at $55^{\circ} \mathrm{C}$ for antigen retrieval. The slides were then permeabilized with $10 \mu \mathrm{g} / \mathrm{ml}$ proteinase $\mathrm{K}$ in $10 \mathrm{mM}$ Tris/ $\mathrm{HCl}(\mathrm{pH}$ 7.6) for $15 \mathrm{~min}$ and incubated with 5\% BSA in a humidified chamber for 30 min to block non-specific binding. The slides were incubated with antibody against NF-kB-p65 (1:200 in PBS containing 1\% BSA; Santa Cruz Biotechnology, Inc.) in a humidified chamber overnight at $4^{\circ} \mathrm{C}$, and with fluorescent-fluorescein isothiocyanate-conjugated donkey antirabbit immunoglobulin G (1:500 in PBS) at room temperature for $1 \mathrm{~h}$. The nuclei were counterstained with Hoechst 33258 $(1: 5,000 ; 10 \mathrm{~min})$. Fluorescence imaging was assessed under a fluorescence microscope (Fluorescence E-1000 microscopy, Nikon, Japan) and subjected to identical exposure times. Instead of the primary antibody, PBS was used for the negative controls.

Statistical analysis. Data were analyzed using SPSS v 11.5 software for Windows (SPSS Inc, Chicago, IL, USA). All values are presented as the means \pm standard error of the mean (SEM). Differences between groups were compared using analysis of variance with the Bonferroni correction for multiple comparisons. Values of $\mathrm{P}<0.05$ were considered to indicate statistically significant differences.

\section{Results}

Osthole improves the survival rate of mice subjected to intestinal I/R injury. In order to determine the effect of osthole on intestinal $\mathrm{I} / \mathrm{R}$ injury, the mice were administered various doses of osthole $(5,10,25,50 \mathrm{mg} / \mathrm{kg})$ for 3 days prior to the induc-

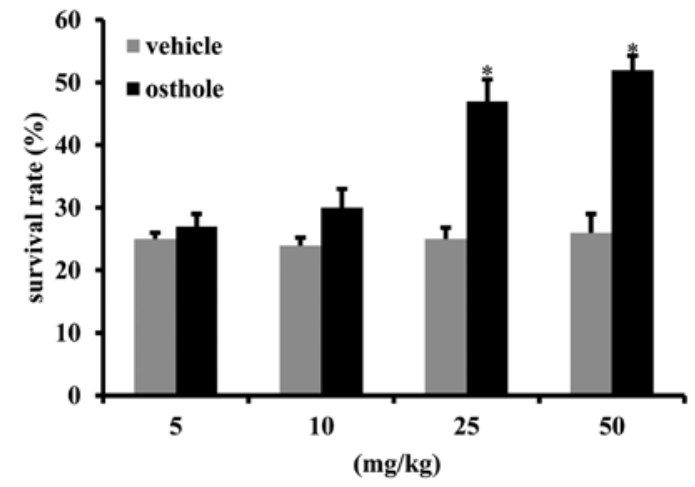

Figure 1. Effects of various doses of osthole on the survival rate of mice subjected to intestinal ischemia/reperfusion (I/R) injury. Mice subjected to intestinal I/R injury were divided into 2 groups. One group $(n=4 \times 9)$ was pretreated with osthole $(5,10,25$ and $50 \mathrm{mg} / \mathrm{kg}$ ) for 3 days and the other group were pre-treated with the vehicle as the control. The final administration of the treatment was $1 \mathrm{~h}$ prior to surgery. The survival rate was calculated at $24 \mathrm{~h}$ after reperfusion. Three independent experiments were performed and data are presented as the means \pm SEM. ${ }^{*} \mathrm{P}<0.05$, statistical significance.

tion of intestinal I/R injury. The results revealed that osthole preconditioning $(25 \mathrm{mg} / \mathrm{kg}$ and $50 \mathrm{mg} / \mathrm{kg}$ ) prior to intestinal I/R injury significantly improved the survival rate at $24 \mathrm{~h}$ following reperfusion as compared to the vehicle-treated mice (Fig. 1).

Osthole attenuates intestinal injury induced by $I / R$. To further investigate the effect of osthole on intestinal I/R injury, the morphology of the intestinal epithelium and villi was examined by immunohistochemistry. In the sham group, the results of histopathological examination of the intestinal epithelium and villi were normal. Mucosal necrosis and bleeding were observed in the intestinal mucosa and submucosa in the $\mathrm{I} / \mathrm{R}$ group which also showed a marked reduction in villus height and destruction of epithelial cells within the villi. In the osthole group, mice pre-treated with osthole $(50 \mathrm{mg} / \mathrm{kg})$ demonstrated intestinal architecture with almost normal histological features. Although the median intestinal injury score of the osthole group was higher than the sham group $(\mathrm{P}<0.001)$, it was significantly lower than that of the $\mathrm{I} / \mathrm{R}$ group $(\mathrm{P}<0.01)$ (Fig. 2B). Taken together, these data suggest that osthole exerts protective effects against intestinal I/R injury.

Osthole exerts antioxidant effects in intestinal $I / R$ injury. To elucidate the underlying mechanisms of action of osthole in protecting against intestinal I/R injury, the antioxidant and oxidant products were evaluated. The levels of SOD, an enzyme occurring naturally in the body which protects cells by cleaning up free radicals, were significantly reduced in the I/R group compared with the sham group $(\mathrm{P}<0.05)$. Following pre-treatment with osthole, SOD activity was significantly improved compared with the I/R group $(\mathrm{P}<0.01)$ (Fig. 3A). The levels of MDA, an important marker of lipoperoxidation associated with oxidative stress, were decreased following pre-treatment with osthole ( $\mathrm{P}<0.05$ vs. I/R group) (Fig. 3B). Following I/R injury, the levels of GSH, a common antioxidant, were slightly decreased; however, following pre-treatment with osthole, these levels increased. However, the difference was not statistically significant among the different groups (Fig. 3C). These results demonstrate that osthole relieves oxidative stress 
A
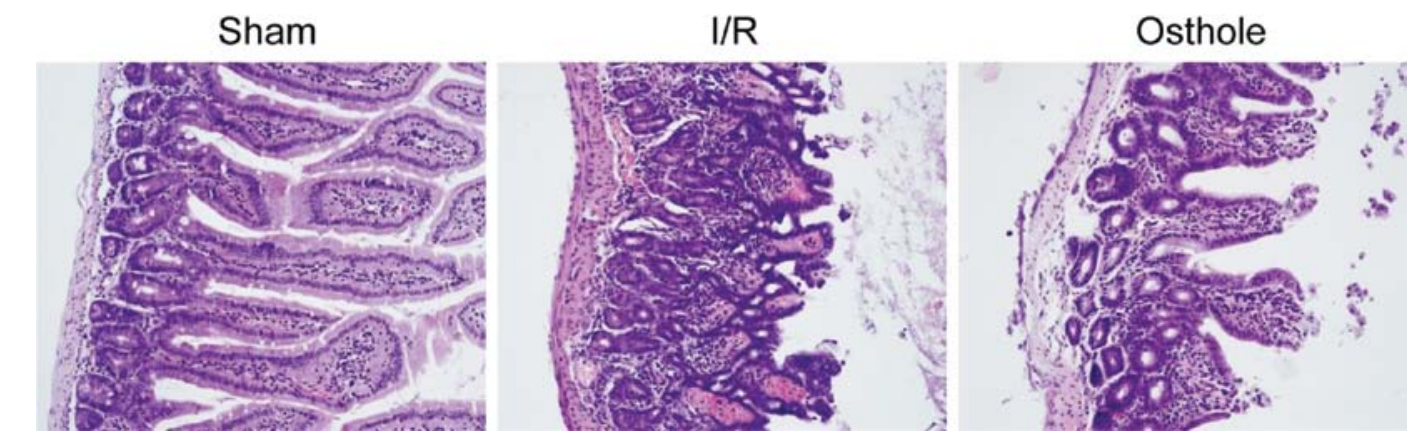

B

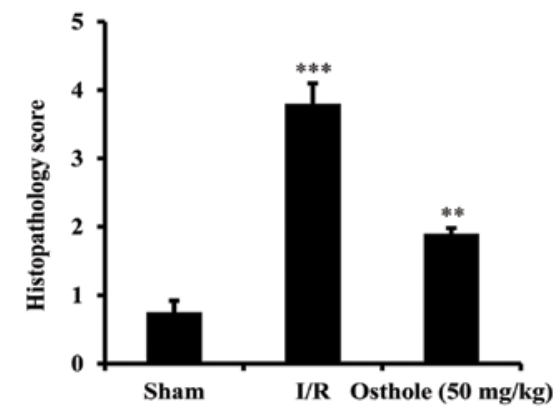

Figure 2. Representative hematoxylin and eosin (H\&E)-stained microscopic images of the intestinal tissue from the different groups. (A) Intestinal segments from the different groups were stained with $\mathrm{H} \& \mathrm{E}$ for histological examination. (B) Histopathology score for intestinal ischemia/reperfusion $(\mathrm{I} / \mathrm{R})$ injury. All mice were randomly allocated into 3 groups. In the sham group, all the surgical steps were performed; however, intestinal I/R was not induced. In the $\mathrm{I} / \mathrm{R}$ group, intestinal I/R was induced and this group served as the control for the osthole-treated group. In the osthole group, the mice subjected to I/R were injected with osthole $(50 \mathrm{mg} / \mathrm{kg})$. All histological analyses were performed by a pathologist in a blinded manner. Five random high-power fields $(\mathrm{x} 200)$ were examined per sample. Data are presented as the means \pm SEM ( $\mathrm{n}=4$ per group). ${ }^{* *} \mathrm{P}<0.01$, statistically significant difference compared with the I/R group; ${ }^{* * *} \mathrm{P}<0.001$, statistically significant difference compared with the sham group.

through the upregulation of SOD and the downregulation of MDA levels following intestinal I/R injury.

Osthole reduces neutrophil infiltration following intestinal $I / R$ injury. Tissue MPO activity is a hallmark of intestinal $I / R$ injury which reflects the infiltration of neutrophils. In order to examine the effect of osthole on inflammation, the activity of MPO was measured. Compared to the sham group, a significant increase in MPO activity was observed in the I/R group, which was alleviated in the osthole-treated group $(\mathrm{P}<0.01)$ (Fig. 4).

Osthole inhibits the elevation of NO levels following intestinal $I / R$ injury. The overproduction of NO following intestinal I/R injury has been reported to be closely associated with oxidative damage (11). NO synthesis can contribute to nitrite accumulation. The concentration of nitrite in the intestinal samples was measured to reflect the production of NO. As shown in Fig. 5, pre-treatment with osthole significantly inhibited the elevation of $\mathrm{NO}$ levels in the $\mathrm{I} / \mathrm{R}$ group $(\mathrm{P}<0.01)$.

Osthole protects against intestinal $I / R$ injury by inhibitig the activation of the $N F-\kappa B$ signaling pathway. It has been reported that NO produced by the iNOS gene is induced during inflammation (24). NF- $\kappa \mathrm{B}$ is an important nuclear
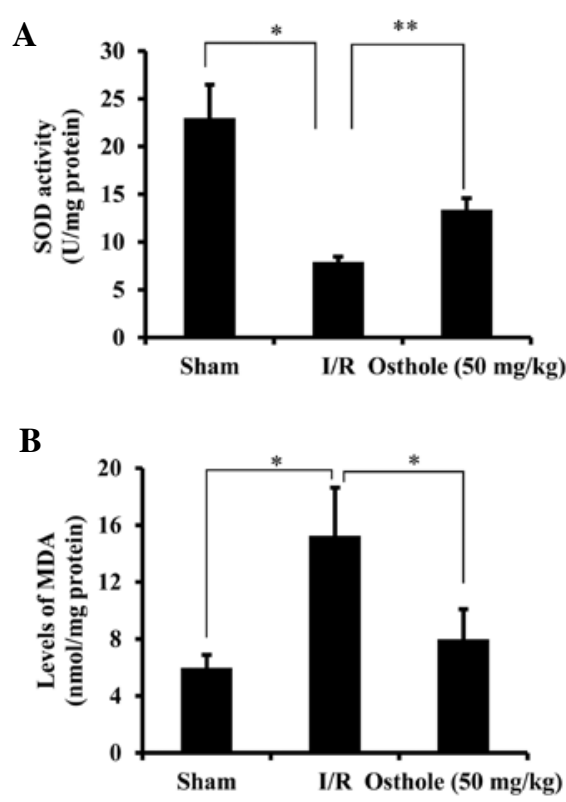

C

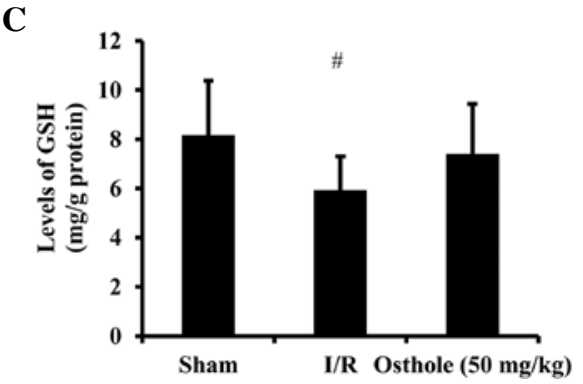

Figure 3. Effects of osthole on antioxidant and oxidant products. (A) Superoxide dismutase (SOD) activity was measured at $500 \mathrm{~nm}$ and expressed as $\mathrm{U} / \mathrm{mg}$ protein. (B) Malondialdehyde (MDA) levels were determined at $532 \mathrm{~nm}$ and the results were expressed as nmol/mg protein. (C) Glutathione (GSH) activity was determined at $420 \mathrm{~nm}$ and the results were expressed as $\mathrm{mg} / \mathrm{g}$ protein Three independent experiments were performed and the data are presented as the means \pm SEM. ${ }^{*} \mathrm{P}<0.05$ and ${ }^{* *} \mathrm{P}<0.01$, statistically significant difference.

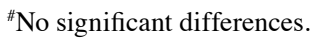

transcription factor that regulates iNOS gene expression by binding the iNOS promoter region and plays a central role in inflammation through its ability to induce the transcription of pro-inflammatory genes (25). The data mentioned above suggest that osthole reduces neutrophil infiltration and inhibits the NO levels induced by intestinal I/R injury. Thus, we hypothesized that osthole protects against intestinal I/R injury by inhibiting the activation of the NF- $\mathrm{kB}$ signaling pathway. To confirm our hypothesis, we examined the effects of osthole on 


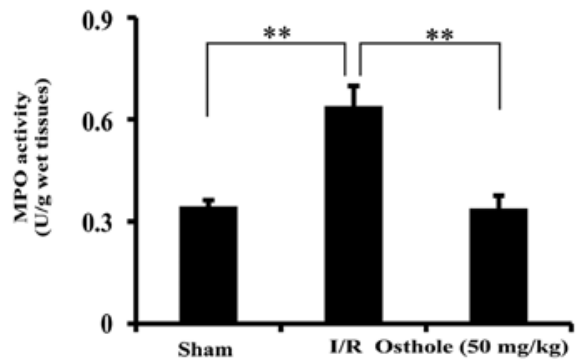

Figure 4. Effects of osthole on myeloperoxidase (MPO) activity. The optical density was recorded at $460 \mathrm{~nm}$ and the results were expressed as $\mathrm{U} / \mathrm{g}$ wet tissue. Three independent experiments were performed and the data are presented as the means \pm SEM. ${ }^{* * *} \mathrm{P}<0.01$, statistically significant difference.

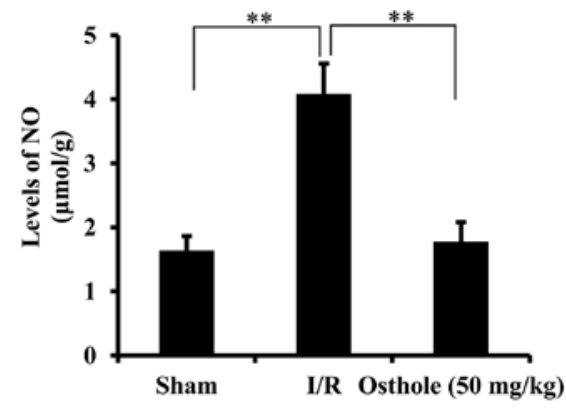

Figure 5. Effects of osthole on nitric oxide (NO) levels. The concentration of nitrite in the intestinal samples was measured following a reaction with Griess reagent (sulfanilamide $1 \%$, naphthylethylene diamine $0.01 \%, \mathrm{H}_{3} \mathrm{PO}_{4} 5 \%$ ) to reflect the production of $\mathrm{NO}$ at $550 \mathrm{~nm}$. The results are expressed as $\mu \mathrm{mol} / \mathrm{g}$ protein. Three independent experiments were performed and the data are presented as the means $\pm \mathrm{SEM} .{ }^{* *} \mathrm{P}<0.01$, statistically significant difference.
$\mathrm{NF}-\kappa \mathrm{B}$ nuclear translocation. In the sham group, NF- $\kappa \mathrm{B}$ was localized in the cytoplasm and translocated to the nucleus in the I/R group. Pre-treatment with osthole inhibited the nuclear translocation of NF- $\mathrm{kB}$ compared with the I/R group (Fig. 6). In order to further confirm these results, western blot analysis was performed to examine the nuclear expression of NF- $\mathrm{KB}$ and the phosphorylation state of $\operatorname{I} \mathrm{B} \alpha$. Consistent with the immunofluorescence staining results, we observed that the nuclear expression of NF- $\mathrm{kB}$ was markedly increased in the I/R group, while pre-treatment with osthole inhibited the nuclear expression of NF- $\mathrm{kB}(\mathrm{P}<0.01 \mathrm{vs}$. I/R group). We also found that phosphorylated I $\kappa \mathrm{B} \alpha$ was increased in the I/R group. In the osthole-treated group, phosphorylated IкB $\alpha$ was significantly inhibited ( $\mathrm{P}<0.01$ vs. I/R group) (Fig. 7).

\section{Discussion}

Osthole, a Chinese herbal medicine possessing a variety of pharmacological properties, has gained considerable attention over the years and has been used for the treatment of a number of diseases, including carcinoma (14), amnesia (26), metabolic syndromes $(27,28)$, seizures (29) and osteoporosis (30). Recent studies have suggested that osthole is also beneficial in preventing I/R injury. It has been shown that osthole prevents motor impairment, decreases the levels of NO, interleukin (IL)- $1 \beta$ and IL-8, inhibits iNOS and MPO activity, and decreases infarct volume following middle cerebral artery occlusion (31). Similarly, it has been demonstrated that osthole significantly inhibits neuronal cell death in the CA1 area in the hippocampus by decreasing caspase- 3 protein expression in

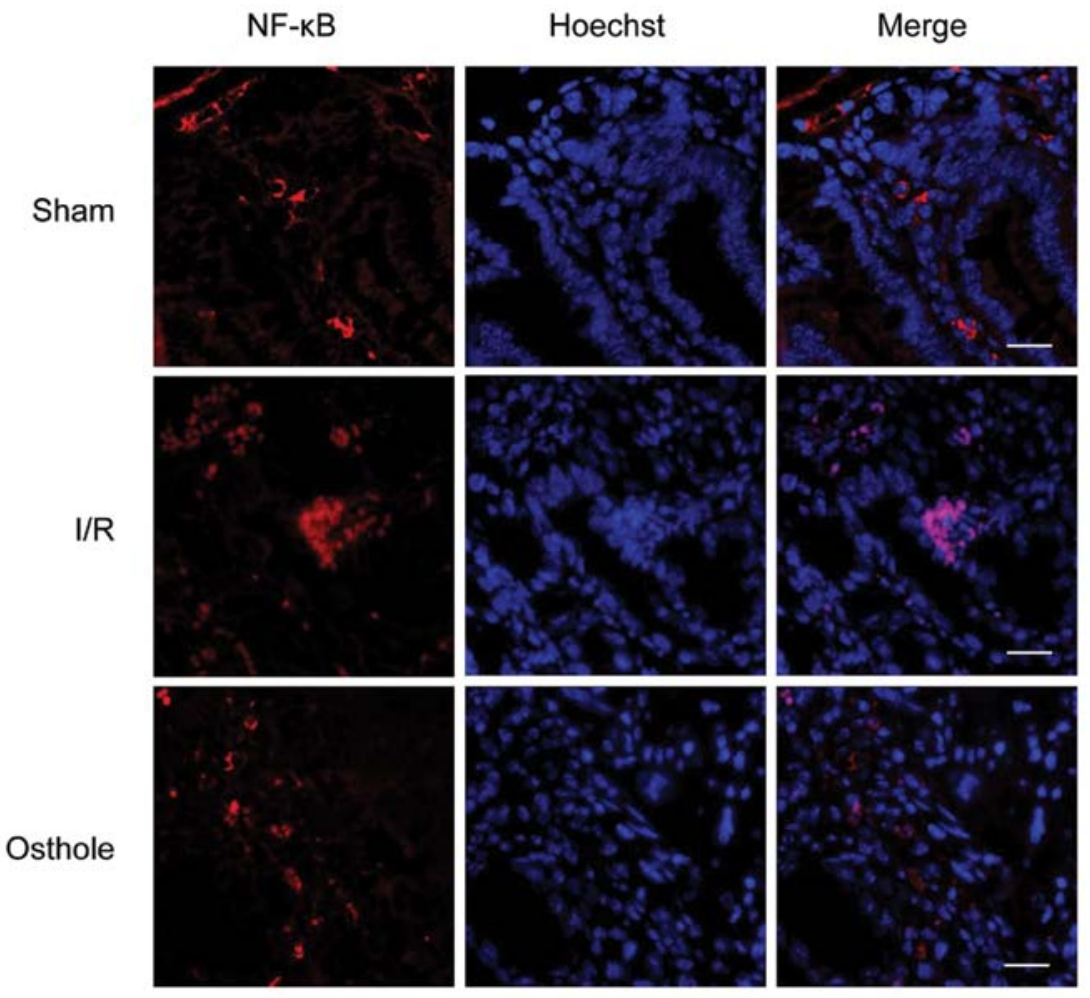

Figure 6. Effects of osthole on the subcellular localization of NF- $\kappa$ B. Detection of NF- $\mathrm{B}$ in intestinal epithelial cells with antibody against NF- $\kappa$ B-p65. Red fluorescence indicates $\mathrm{NF}-\kappa \mathrm{B}$. Blue fluorescence indicates the nucleus stained with Hoechst 33258 . The pink color is caused by the overlap of red-colored $\mathrm{NF}-\kappa \mathrm{B}$ and bule-colored nucleus. 
A

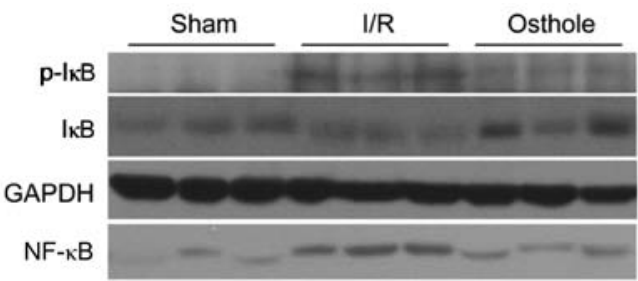

B

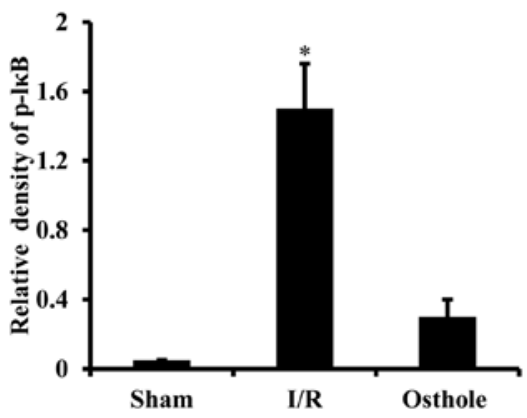

C

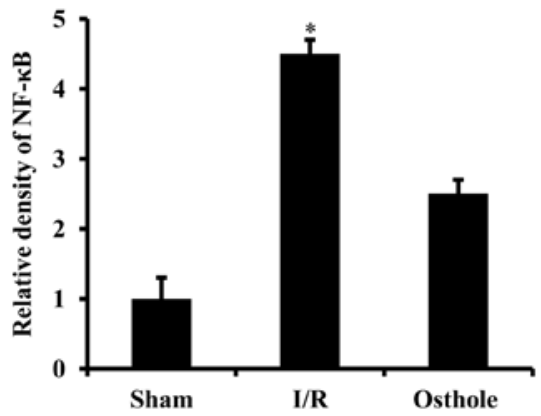

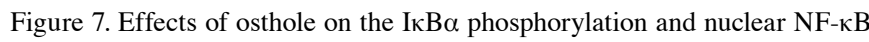
expression. (A) Western blot analysis revealed that the levels of $\mathrm{p}-\mathrm{I} \kappa \mathrm{B} \alpha$ protein were higher in the ischemia/reperfusion (I/R) group compared to the sham group. Osthole significantly inhibited IкB $\alpha$ phosphorylation. An enhanced expression of the NF-kB p65 subunit was observed in the I/R group, while pre-treatment with osthole inhibited the nuclear expression of the NF-kB p65 subunit. GAPDH was used as the loading control. (B and C) The protein expression was analyzed using BandScan 5.0 software and normalized to GAPDH The data are presented as the means \pm SEM for 3 samples and 3 independent experiments were performed. ${ }^{*} \mathrm{P}<0.05$, statistically significant difference.

male SD rats subjected to transient global brain ischemia (32). However, no studies have been conducted on the role and potential mechanisms of action of osthole in intestinal I/R injury. In the present study, we demonstrated that pre-treatment with osthole exerted protective effects against intestinal I/R injury. Pre-treatment with osthole improved the survival rates of mice subjected to I/R injury and protected intestinal epithelial cells and villi against I/R injury. Osthole also suppressed stress, neutrophil infiltration and NO overproduction. Furthermore, we found that the underlying mechanisms of action of osthole in preventing intestinal $\mathrm{I} / \mathrm{R}$ injury involved the inhibition of $\mathrm{NF}-\kappa \mathrm{B}$.

The production of free radicals from oxygen molecules derived from the electron transport chains of mitochondrial cells, endothelial cells and activated neutrophils elicits oxidative stress and enhances intestinal I/R injury (33). Thus, the antioxidant, $\mathrm{SOD}$, is considered to play a crucial role in relieving oxidative stress during I/R injury. In this study, we found that pre-treatment with osthole upregulated SOD activity which was suppressed in the I/R group. Osthole also downregulated MDA levels following intestinal I/R injury, which are a marker of lipoperoxidation associated with oxidative stress. These results are in agreement with those from previous reports demonstrating that osthole is effective in treating hyperlipidemic and alcoholic fatty liver by the inhibition of ROS production, the enhancement of antioxidative enzyme activity and the reduction of peroxidation $(34,35)$. In addition, it was found that the neuroprotective effects of osthole against acute ischemic stroke and 1-methyl-4-phenylpyridinium ion-induced cytotoxicity in PC12 cells is partly attributed to its antioxidative action (36). Neutrophil infiltration has been proposed as a hallmark of intestinal I/R injury. The activity of MPO which is mainly released by neutrophils can be evaluated as neutrophil infiltration (11). In our study, MPO activity was significantly augmented in the I/R group and was decreased following pre-treatment with osthole. Furthermore, the involvement of $\mathrm{NO}$ in intestinal I/R injury has been widely suggested. iNOS can produce excessive amounts of NO following intestinal I/R injury, which is considered responsible for the cytotoxic potential of $\mathrm{NO}$, resulting in oxidative stress and tissue damage $(11,37,38)$. A previous study demonstrated that osthole decreased NO levels and inhibited NOS activity in a rat model of carrageenan-induced hind paw edema (17). In the current study, we found that osthole markedly inhibited the increased NO levels induced by intestinal I/R injury. These results suggest that osthole exerts therapeutic effects against intestinal I/R injury by attenuating oxidative stress, reducing excessive neutrophil infiltration and modulating NO levels.

However, the mechanisms of action of osthole in exerting its protective effects remain to be elucidated. The iNOS promoter region contains binding sites for $\mathrm{NF}-\kappa \mathrm{B}$, and its expression during $\mathrm{I} / \mathrm{R}$ injury requires de novo transcription, which is under the control of $\mathrm{NF}-\kappa \mathrm{B}$. NF- $\kappa \mathrm{B}$ is usually present in the cytoplasm in an inactive form bound to the inhibitory protein, $\mathrm{I}-\kappa \mathrm{B} . \mathrm{NF}-\kappa \mathrm{B}$ is activated by a variety of stimuli, including growth factors, cytokines, oxidative stress and proinflammatory stimuli, and the nuclear translocation of NF- $\kappa \mathrm{B}$ subunits is induced following intestinal I/R injury $(39,40)$. It has been reported that iNOS is capable of producing abundant NO under certain pathological conditions, which presents beneficial effects but also contributes to the overproduction of cytotoxic radicals (41). In a previous study, it was shown that osthole, isolated from Clausena guillauminii, inhibited the protein expression of iNOS induced by LPS in mouse macrophages (42). Another study also demonstrated that osthole reduced tumor necrosis factor (TNF)- $\alpha$, NO and cyclooxygenase (COX)-2 expression, inhibited $\mathrm{NF}-\kappa \mathrm{B}$ activation and ROS release in LPS-stimulated macrophages (43). In this study, pre-treatment with osthole inhibited the translocation of $\mathrm{NF}-\kappa \mathrm{B}$ to the nucleus and increased $\mathrm{I}-\kappa \mathrm{B}$ phosphorylation induced by intestinal I/R injury. These data suggest that osthole exerts protective effects against intestinal I/R injury through the NF- $\kappa \mathrm{B}-\mathrm{iNOS}-\mathrm{NO}$ pathway. Together with the free radical scavenging and anti-inflammatory effects, osthole may be a potential candidate for the development of anti-ischemic drugs.

Certain limitations of this study should be noted. Intestinal IR injury has been extensively investigated in animal models and various approaches to diminish the damaging effects of intestinal I/R injury have been investigated in animal models, 
as well as in vitro $(44,45)$. However, these models are not a realistic model of the clinical situation and there are a number of obvious differences between animal models and humans during intestinal I/R injury, including antigens of specific proteins and sensitivity to intestinal ischemia and inflammatory responses (46-48). Moreover, advances in the clinical treatment of I/R injury have been minimal. Pre-teatment with osthole prior to ischemia in this study may not be a realistic model of the clinical situation. The mice were pre-treated with osthole prior to the induction of ischemia. Additionally, it was confirmed that osthole preconditioning relieved cerebral ischemic stroke (19). Therefore, although a recent study confirmed that treatment with osthole subsequent to ischemia significantly improved the cognitive deficits induced by chronic cerebral hypoperfusion in rats (49), it can only be speculated whether or not osthole postconditioning would also attenuate intestinal I/R injury. To clarify this important point, treatment with osthole at different starting points following the onset of intestinal ischemia or reperfusion should be investigated.

Taken together, the data from our study demonstrate that pre-treatment with osthole attenuates oxidative stress, reduces neutrophil infiltration and decreases NO levels. The mechanisms behind the protective effects of osthole involve the

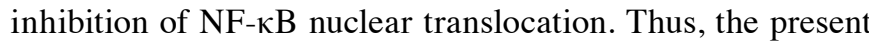
study suggests that osthole exerts protective and therapeutic effects against intestinal I/R injury.

\section{Acknowledgements}

This study was supported by the National Natural Science Foundation of Cultivating Projects of the First Affiliated Hospital of Anhui Medical University (grant no. 2009KJ12) and the Anhui Province Natural Science Foundation (grant no. 11040606Q21). The authors would like to thank Dr Xiao-Dong Cao and Dr Wen-Bo Liu for their technical assistance.

\section{References}

1. Yasuhara H: Acute mesenteric ischemia: the challenge of gastroenterology. Surg Today 35: 185-195, 2005.

2. Berland T and Oldenburg WA: Acute mesenteric ischemia. Curr Gastroenterol Rep 10: 341-346, 2008.

3. Ozacmak VH, Sayan H, Arslan SO, Altaner S and Aktas RG: Protective effect of melatonin on contractile activity and oxidative injury induced by ischemia and reperfusion of rat ileum. Life Sci 76: 1575-1588, 2005.

4. Shibata C, Balsiger BM, Anding WJ, Duenes JA, Miller VM and Sarr MG: Functional changes in nonadrenergic, noncholinergic inhibitory neurons in ileal circular smooth muscle after small bowel transplantation in rats. Dig Dis Sci 43: 2446-2454, 1998.

5. Blikslager AT, Moeser AJ, Gookin JL, Jones SL and Odle J: Restoration of barrier function in injured intestinal mucosa. Physiol Rev 87: 545-564, 2007.

6. Szabo A, Vollmar B, Boros M and Menger MD: In vivo fluorescence microscopic imaging for dynamic quantitative assessment of intestinal mucosa permeability in mice. J Surg Res 145 179-185, 2008.

7. Santora RJ, Lie ML, Grigoryev DN, Nasir O, Moore FA and Hassoun HT: Therapeutic distant organ effects of regional hypothermia during mesenteric ischemia-reperfusion injury. J Vasc Surg 52: 1003-1014, 2010

8. Vasileiou I, Kostopanagiotou G, Katsargyris A, Klonaris C, Perrea D and Theocharis S: Toll-like receptors: a novel target for therapeutic intervention in intestinal and hepatic ischemiareperfusion injury? Expert Opin Ther Targets 14: 839-853, 2010.
9. Shah PC, Brolin RE, Amenta PS and Deshmukh DR: Effect of aging on intestinal ischemia and reperfusion injury. Mech Ageing Dev 107: 37-50, 1999.

10. Esposito E and Cuzzocrea S: Role of nitroso radicals as drug targets in circulatory shock. Br J Pharmacol 157: 494-508, 2009.

11. Barocelli E, Ballabeni V, Ghizzardi P, et al: The selective inhibition of inducible nitric oxide synthase prevents intestinal ischemiareperfusion injury in mice. Nitric Oxide 14: 212-218, 2006.

12. Nakano H, Nakajima A, Sakon-Komazawa S, Piao JH, Xue X and Okumura K: Reactive oxygen species mediate crosstalk between NF-kappaB and JNK. Cell Death Differ 13: 730-737, 2006.

13. Hoult JR and Paya M: Pharmacological and biochemical actions of simple coumarins: natural products with therapeutic potential. Gen Pharmacol 27: 713-722, 1996.

14. Chou SY, Hsu CS, Wang KT, Wang MC and Wang CC: Antitumor effects of osthole from Cnidium monnieri: an in vitro and in vivo study. Phytother Res 21: 226-230, 2007.

15. Zhang Q, Qin L, He W, et al: Coumarins from Cnidium monnieri and their antiosteoporotic activity. Planta Med 73: 13-19, 2007.

16. Liang HJ, Suk FM, Wang CK, et al: Osthole, a potential antidiabetic agent, alleviates hyperglycemia in $\mathrm{db} / \mathrm{db}$ mice. Chem Biol Interact 181: 309-315, 2009.

17. Liu J, Zhang W, Zhou L, Wang X and Lian Q: Anti-inflammatory effect and mechanism of osthole in rats. Zhong Yao Cai 28: 1002-1006, 2005

18. Chiu PR, Lee WT, Chu YT, Lee MS, Jong YJ and Hung CH: Effect of the Chinese herb extract osthole on IL-4-induced eotaxin expression in BEAS-2B cells. Pediatr Neonatol 49: $135-140,2008$

19. Chao X, Zhou J, Chen T, et al: Neuroprotective effect of osthole against acute ischemic stroke on middle cerebral ischemia occlusion in rats. Brain Res 1363: 206-211, 2010.

20. Li F, Gong Q, Wang L and Shi J: Osthole attenuates focal inflammatory reaction following permanent middle cerebral artery occlusion in rats. Biol Pharm Bull 35: 1686-1690, 2012.

21. Zhou F, Zhong W, Xue J, Gu ZL and Xie ML: Reduction of rat cardiac hypertrophy by osthole is related to regulation of cardiac oxidative stress and lipid metabolism. Lipids 47: 987-994, 2012.

22. Souza DG, Soares AC, Pinho V, et al: Increased mortality and inflammation in tumor necrosis factor-stimulated gene-14 transgenic mice after ischemia and reperfusion injury. Am J Pathol 160: 1755-1765.

23. Fleming SD, Monestier $M$ and Tsokos GC: Accelerated ischemia/reperfusion-induced injury in autoimmunity-prone mice. J Immunol 173: 4230-4235, 2004.

24. Nathan C: Nitric oxide as a secretory product of mammalian cells. FASEB J 6: 3051-3064, 1992.

25. Tak PP and Firestein GS: NF-kappaB: a key role in inflammatory diseases. J Clin Invest 107: 7-11, 2001.

26. Hsieh MT, Hsieh CL, Wang WH, Chen CS, Lin CJ and Wu CR: Osthole improves aspects of spatial performance in ovariectomized rats. Am J Chin Med 32: 11-20, 2004.

27. Zhang Y, Xie ML, Zhu LJ and Gu ZL: Therapeutic effect of osthole on hyperlipidemic fatty liver in rats. Acta Pharmacol Sin 28: 398-403, 2007.

28. Zhang Y, Xie ML, Xue J and Gu ZL: Osthole regulates enzyme protein expression of CYP7A1 and DGAT2 via activation of PPARalpha/gamma in fat milk-induced fatty liver rats. J Asian Nat Prod Res 10: 807-812, 2008.

29. Luszczki JJ, Andres-Mach M, Cisowski W, Mazol I, Glowniak K and Czuczwar SJ: Osthole suppresses seizures in the mouse maximal electroshock seizure model. Eur J Pharmacol 607: 107-109, 2009.

30. Li XX, Hara I and Matsumiya T: Effects of osthole on postmenopausal osteoporosis using ovariectomized rats; comparison to the effects of estradiol. Biol Pharm Bull 25: 738-742, 2002.

31. He W, Liu JX, Zhou YM, et al: Protective effects of osthole on cerebral ischemia-reperfusion injury in rats and its mechanism. Chin Pharmacol Bull 24: 1528-1530, 2008.

32. Liu WB, Huo JL, Fei Z, Men XL and Li J: Neuroprotection of osthole against transient global brain ischemia in rats. Chin $\mathrm{J}$ Neurosurg Dis Res 8: 118-121, 2009.

33. Flessas II,Papalois AE, Toutouzas K, Zagouri F and Zografos GC: Effects of lazaroids on intestinal ischemia and reperfusion injury in experimental models. J Surg Res 166: 265-274, 2009.

34. Song F, Xie ML, Zhu LJ, Zhang KP, Xue J and Gu ZL: Experimental study of osthole on treatment of hyperlipidemic and alcoholic fatty liver in animals. World $\mathbf{J}$ Gastroenterol 12: 4359-4363, 2006. 
35. Sun F, Xie ML, Xue J and Wang HB: Osthole regulates hepatic PPAR alpha-mediated lipogenic gene expression in alcoholic fatty liver murine. Phytomedicine 17: 669-673, 2010.

36. Liu WB, Zhou J, Qu Y, et al: Neuroprotective effect of osthole on $\mathrm{MPP}^{+}$-induced cytotoxicity in PC12 cells via inhibition of mitochondrial dysfunction and ROS production. Neurochem Int 57: 206-215, 2010.

37. Naito $\mathrm{Y}$, Takagi $\mathrm{T}$, Ichikawa $\mathrm{H}$, et al: A novel potent inhibitor of inducible nitric oxide inhibitor, ONO-1714, reduces intestinal ischemia-reperfusion injury in rats. Nitric Oxide 10: 170-177, 2004

38. Suzuki Y, Deitch EA, Mishima S, Lu Q and Xu D: Inducible nitric oxide synthase gene knockout mice have increased resistance to gut injury and bacterial translocation after an intestinal ischemia-reperfusion injury. Crit Care Med 28: 3692-3696, 2000.

39. Zou L, Attuwaybi B and Kone BC: Effects of NF-kappa B inhibition on mesenteric ischemia-reperfusion injury. Am J Physiol Gastrointest Liver Physiol 284: G713-G721, 2003.

40. Souza DG, Vieira AT, Pinho V, et al: NF-kappaB plays a major role during the systemic and local acute inflammatory response following intestinal reperfusion injury. Br J Pharmacol 145: 246-254, 2005

41. Altavilla D, Squadrito F, Campo GM, et al: The lazaroid, U-74389G, inhibits inducible nitric oxide synthase activity, reverses vascular failure and protects against endotoxin shock. Eur J Pharmacol 369: 49-55, 1999.

42. Nakamura T, Kodama N, Arai Y, et al: Inhibitory effect of oxycoumarins isolated from the Thai medicinal plant Clausena guillauminii on the inflammation mediators, iNOS, TNF-alpha, and COX-2 expression in mouse macrophage RAW 264.7. J Nat Med 63: 21-27, 2009.
43. Liao PC, Chien SC, Ho CL, et al: Osthole regulates inflammatory mediator expression through modulating NF-kappaB, mitogenactivated protein kinases, protein kinase $\mathrm{C}$, and reactive oxygen species. J Agric Food Chem 58: 10445-10451, 2010.

44. Duggan M, Engelberts D, Jankov RP, et al: Hypocapnia attenuates mesenteric ischemia-reperfusion injury in a rat model. Can $\mathrm{J}$ Anaesth 52: 262-268, 2005.

45. Spanos CP, Papaconstantinou P, Spanos P, Karamouzis M Lekkas G and Papaconstantinou C: The effect of L-arginine and aprotinin on intestinal ischemia-reperfusion injury. J Gastrointest Surg 11: 247-255, 2007.

46. Bjorck M, Bergqvist D, Rasmussen I, Piehl E and Haglund U: An experimental porcine model of partial ischaemia of the distal colon. Eur J Surg 163: 843-850, 1997.

47. Grootjans J, Lenaerts K, Derikx JP, et al: Human intestinal ischemia-reperfusion-induced inflammation characterized: experiences from a new translational model. Am J Pathol 176: 2283-2291, 2010

48. Robinson JW, Mirkovitch V, Winistorfer B and Saegesser F: Response of the intestinal mucosa to ischaemia. Gut 22: 512-527, 1981.

49. Ji HJ, Hu JF, Wang YH, Chen XY, Zhou R and Chen NH: Osthole improves chronic cerebral hypoperfusion induced cognitive deficits and neuronal damage in hippocampus. Eur J Pharmacol 636: 96-101, 2010 